\title{
The submm wave Josephson flux flow oscillator; Linewidth measurements and simple theory
}

Mygind, Jesper; Koshelets, V. P.; Samuelsen, Mogens Rugholm; Sobolev, Alexander

Published in:

I E E E Transactions on Applied Superconductivity

Link to article, DOI:

10.1109/TASC.2005.850143

Publication date:

2005

Document Version

Publisher's PDF, also known as Version of record

Link back to DTU Orbit

Citation (APA):

Mygind, J., Koshelets, V. P., Samuelsen, M. R., \& Sobolev, A. (2005). The submm wave Josephson flux flow oscillator; Linewidth measurements and simple theory. I E E E Transactions on Applied Superconductivity, 15(2), 968-971. https://doi.org/10.1109/TASC.2005.850143

\section{General rights}

Copyright and moral rights for the publications made accessible in the public portal are retained by the authors and/or other copyright owners and it is a condition of accessing publications that users recognise and abide by the legal requirements associated with these rights.

- Users may download and print one copy of any publication from the public portal for the purpose of private study or research.

- You may not further distribute the material or use it for any profit-making activity or commercial gain

- You may freely distribute the URL identifying the publication in the public portal 


\title{
The Submm Wave Josephson Flux Flow Oscillator; Linewidth Measurements and Simple Theory
}

\author{
J. Mygind, V. P. Koshelets, M. R. Samuelsen, and A. S. Sobolev
}

\begin{abstract}
The Flux Flow Oscillator (FFO) is a long Josephson junction in which a DC bias current and a DC magnetic field maintain a unidirectional viscous flow of magnetic flux quanta. The theoretical linewidth of the electromagnetic radiation generated at the end boundary is due to internal current fluctuations as given by the usual expression for the lumped junction. Experimentally, however, the linewidth deviates significantly both in magnitude and functional dependence. We suggest a simple solution based on the assumption that the bias current creates an additional magnetic field in the junction. This is supported by linewidth measurements on FFO's subjected to different bias configurations.
\end{abstract}

Index Terms-Josephson junctions, submillimeter wave oscillators, superconducting devices.

\section{INTRODUCTION}

$\mathbf{T}$ HEORETICALLY the spectral linewidth $\Delta \nu$ (full width half power) of the lumped Josephson junction oscillator is given by [1]

$$
\Delta \nu=\pi \frac{R_{d}^{2}}{\Phi_{0}^{2}} S_{I}(0)
$$

where $R_{d}$ is the dynamic resistance in the bias point and $\Phi_{0}=$ $h / 2 e$ is the flux quantum. $S_{I}(0)$ is the power density of the internal low frequency current fluctuations including both thermal noise and shot noise [1]

$$
S_{I}(0)=2 e\left\{I_{q p} \operatorname{coth} v+2 I_{s} \operatorname{coth} 2 v\right\} \quad \text { with } \quad v=\frac{e V}{2 k_{B} T},
$$

where $I_{q p}$ is the DC quasiparticle current and $I_{s}$ is the DC superconducting pair current. $T$ is the physical temperature. Eq. (1) comes from standard frequency modulation theory and the terms $R_{d}^{2}$ and $\phi_{0}^{2}$ originate in the basic transformation of a current noise power spectrum to a voltage spectrum, and from the voltage spectrum to a frequency spectrum, respectively. The pair current term accounts for the fact that the junction was coupled to a lossy resonator [1]. Eq. (2) was derived for a tunnel junction DC biased at voltage $V$ but a similar formula may be obtained for an arbitrary bias source [2]. Deviations from the assumed white noise spectrum may be included as an effective temperature, $T_{\text {eff }}>T$.

Manuscript received October 5, 2004. The work was supported in part by the RFBR project 03-02-16748, INTAS project 01-0367, ISTC project 2445 , the Danish Natural Science Foundation and the Hartmann Foundation.

J. Mygind and M. R. Samuelsen are with the Department of Physics, B309, Technical University of Denmark, DK-2800 Lyngby, Denmark (e-mail: myg@fysik.dtu.dk).

V. P. Koshelets and A. S. Sobolev are with the Institute of Radio Engineering and Electronics (IREE), Moscow, Russia (e-mail: valery@hitec.cplire.ru).

Digital Object Identifier 10.1109/TASC.2005.850143
Generally (1) applies to most high- $T_{c}$ and low- $T_{c}$ Josephson oscillators e.g. point contacts and micro bridges, short tunnel junctions, and resonant fluxon oscillators [3]. For wellcharacterized small tunnel junctions an observed disagreement (pre-factor $\approx 2$ ) between experiment and theory can be accounted for by a modified current noise spectrum (quantum effects). For some metallic weak links and high- $T_{c}$ junctions larger discrepancies (pre-factors of 2 to 100) have been observed. The resonant fluxon (soliton) oscillator has an extra pre-factor $1 / 4$ because the fluxon-antifluxon reflection at each boundary results in a $4 \pi$ change of the phase difference and thus to a modified Josephson frequency-phase relation. All these Josephson oscillators need only a single DC bias, usually supplied from a current source.

The Flux Flow Oscillator (FFO) differs from the other members of the Josephson oscillator family by needing also an external DC magnetic field e.g. from a current in a "control" line. Theoretically it has been shown [4], [5] that the linewidth of an ideal FFO (i.e. with perfect overlap geometry and constant bias current distribution) is also given by the lumped junction expression (1), (2). However, experimentally there is a substantial discrepancy (up to a factor 10) between the linewidth of real FFO's and that calculated using (1). Also a different functional dependence on $R_{d}$ is found. This has been a puzzling problem for almost a decade [4]-[8]. In order to remedy the functional dependence and to obtain agreement with (1), (2) it was first tried empirically [9], [10] to include noise related to the control line current. Although the procedure provides good fit to our experiments, there is no theoretical justification for it. In fact, the implicit assumption [10] that noise in the junction current and noise in the control line current are fully correlated, implies that they both originate in the internal current fluctuations.

\section{Sine-Gordon Model With Boundaries}

In a simple picture the dynamics of the FFO is a unidirectional viscous flow of mutually repulsive fluxons in a long Josephson junction. The DC bias current, $I$, drives the fluxon chain while the applied DC magnetic field from a current, $I_{c l}$, regulates the distance between the fluxons. Experimentally, $I_{c l}$ flows in an integrated overlaying "control" line, in one of the junction electrodes, or in an external coil placed near the junction. The average number of fluxons passing per second gives the DC voltage, $V$, across the junction. Experimentally both $I$ and $I_{c l}$ may be used to tune the junction voltage and thus the frequency of the FFO. Electromagnetic power may be extracted from the end of the junction where the fluxon chain collides with the boundary. In the frequency range $100-700 \mathrm{GHz}$ it has been demonstrated that a standard $\mathrm{Nb} / \mathrm{Al}_{2} \mathrm{O}_{3} / \mathrm{Nb}$ FFO can produce about $1 \mu \mathrm{W}$, which is sufficient to pump an SIS mixer integrated on the same chip [11]. 
Using normalized units a long (length $l$ ) and narrow (width $w$ ) rectangular Josephson junction biased from a DC current supply is well modeled by the one-dimensional $(l \gg w, w \ll 1)$ perturbed sine-Gordon equation [3] $\phi_{x x}-\phi_{t t}=\sin \phi+\alpha \phi_{t}-\eta$ for the phase difference $\phi(x, t)$ across the junction. The normalized overlap current through the junction is $\eta$ and $\alpha$ is the normalized damping. Time $t$ is normalized to the inverse maximum plasma frequency, $\omega_{0}$, length $x$ to the Josephson penetration length, $\lambda_{J}$, currents to the maximum critical current, $I_{c}$, and magnetic field to $J_{c} \lambda_{J}$, where $J_{c}$ is the critical current density, and $H_{c}=2 J_{c} \lambda_{J}$ is the critical field needed to force the first fluxon into the junction.

The normalized magnetic field $\kappa_{1,2}$ at the two ends of the junction enters as the boundary condition

$$
\phi_{x}(0, t)=\kappa_{1} \quad \text { and } \quad \phi_{x}(l, t)=\kappa_{2} .
$$

We assume that the field is in the plane of the junction and perpendicular to the $x$-direction. The total normalized current through the junction is

$$
i=i_{o v}+i_{i n}=w\left(\eta l+\kappa_{2}-\kappa_{1}\right),
$$

where $i_{o v}=\eta w l=\int_{0}^{l} w(x) \eta(x) d x$ is the normalized overlap current, $\left(\kappa_{2}-\kappa_{1}\right) w=i_{\text {in }}$ is the inline part of the normalized junction current, and

$$
\kappa=\frac{\left(\kappa_{1}+\kappa_{2}\right)}{2}
$$

is the normalized magnetic field. The overlap fraction of the junction current is [12]

$$
\chi=\frac{i_{o v}}{i_{i n}+i_{o v}} .
$$

The notation overlap and inline refers to the two idealized geometries for the long rectangular junction, where the DC bias current enters and leaves via the two long boundaries, or via the two narrow end boundaries, respectively.

The normalized DC $I-V$ curve is given by

$$
\omega=\omega\left(\eta, \kappa_{1}, \kappa_{2}\right)=\omega(i, \kappa),
$$

where $\omega=\left\langle\phi_{t}\right\rangle$ is the time average voltage across the junction. The $I-V$ curve of a long low-damped overlap junction with homogeneous current distribution ( $\chi=1, \eta(x)$ constant) exhibits a very distinct step structure [13] with small dynamic resistance. Generally, both an inhomogeneous overlap current distribution $\eta(x)$ and/or some additional inline current supply $(\chi<1)$ will alter the appearance of the structure. Higher damping broadens the structure and eventually turns it into the so-called flux flow step (FFS). For fixed bias current the voltage of the FFS is proportional to the magnetic field [13].

Until now we have discussed an ideal ("bare") junction where $i$ and $\kappa$ are independent variables with $i$ proportional to the externally applied DC bias current $I$ and $\kappa$ proportional to the DC current $I_{c l}$ in the control line. For later use we define two normalized dynamic resistances $r_{d}$ and $r_{d}^{\kappa}$ as partial derivatives of (7) with respect to the bias and control line current, respectively;

$$
r_{d}=\frac{\partial \omega}{\partial i}, \quad r_{d}^{\kappa}=\frac{\partial \omega}{\partial \kappa} \frac{1}{w},
$$

where the dynamic resistance $r_{d}^{\kappa}$ is derived from the current $w \kappa$ equivalent to the magnetic field $\kappa$. The dynamic resistance, $r_{d}$, inserted (in unnormalized units) in (1) gives the linewidth of the "bare" FFO [4], [5].

\section{Magnetic Field Generated by the Bias CuRRent}

Now we consider the case where also the DC bias current generates a magnetic field in the junction. We assume that the normalized magnetic field in the junction consists of two contributions, an externally applied field: $\kappa_{a p p l}=\beta i_{c l}(1 / w)$ proportional to a DC current, $i_{c l}$, in the control line, and a field: $-\sigma i$ proportional to the external DC bias current, $i$. As exemplified below the latter may be due to asymmetry of the junction and the way the bias current is fed to the junction.

$$
\kappa w=\kappa_{a p p l} w-\sigma i=\beta i_{c l}-\sigma i .
$$

Here $\beta$ and $\sigma$ are dimensionless factors determined by junction geometry and bias conditions. Now the measured normalized $I-V$ curve is

$$
\omega=\omega\left(i, \beta i_{c l}-\sigma i\right),
$$

and correspondingly the measured normalized dynamical resistance is

$$
r_{d}^{\prime}=\left.\frac{d \omega}{d i}\right|_{i_{c l}}=\frac{\partial \omega}{\partial i}+\frac{\partial \omega}{\partial \kappa} \frac{1}{w}(-\sigma)=r_{d}-\sigma r_{d}^{\kappa} .
$$

We define a normalized control line dynamical resistance as

$$
r_{d}^{c l}=\left.\frac{d \omega}{d i_{c l}}\right|_{i}=\frac{\partial \omega}{\partial \kappa} \frac{1}{w} \beta=\beta r_{d}^{\kappa} .
$$

i.e. the measured control line dynamical resistance $\left(r_{d}^{c l}\right)^{\prime}$ is the same as before $\left(r_{d}^{c l}\right)^{\prime}=r_{d}^{c l}$. The normalized dynamic resistance, $r_{d}$, entering the linewidth expression (1) for the "bare" FFO is related to the measured dynamic resistances by

$$
r_{d}=r_{d}^{\prime}+\frac{\sigma}{\beta}\left(r_{d}^{c l}\right)^{\prime}=r_{d}^{\prime}+K\left(r_{d}^{c l}\right)^{\prime},
$$

where $K=\sigma / \beta$ is the ratio between the two geometrical factors; $\sigma$ for the external bias current and $\beta$ for the external control line current. If the measured dynamical resistances as introduced in (13) are returned (in unnormalized units) to (1) the linewidth expression is replaced by

$$
\Delta \nu=\pi \frac{\left(R_{d}^{\prime}+K R_{d}^{c l}\right)^{2}}{\phi_{0}^{2}} S_{I}(0),
$$

which contains just the empirical correction factor $\left(R_{d}^{\prime}+K R_{d}^{c l}\right)^{2}$ used by Koshelets et al. [9] instead of $\left(R_{d}\right)^{2}$ to obtain a good fit to (1), (2) with $K$ as fitting parameter. With their particular junction layout the best fit was achieved for $K \simeq 1$.

All quantities in (14) can be measured with good accuracy. In a given bias point the free-running linewidth is measured with the FFO frequency-locked (by a frequency discriminator circuit) to a $400 \mathrm{MHz}$ reference using an on-chip integrated SIS junction as external harmonic mixer to down-convert the $100-700 \mathrm{GHz}$ signal so that it can be recorded with a spectrum analyzer [8]. The corresponding two measured dynamical resistances $\left(R_{d}\right)^{\prime}=\Delta V / \Delta I$ and $\left(R_{d}^{c l}\right)^{\prime}=\left(R_{d}^{c l}\right)=\Delta V / \Delta I_{c l}$ are calculated from the small voltage change $\Delta V$ found when we 


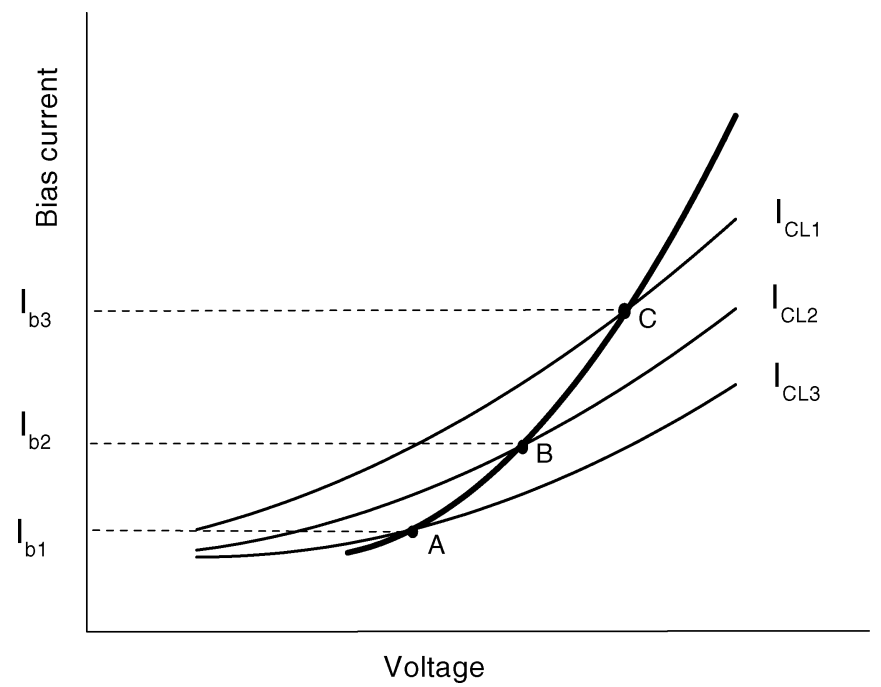

Fig. 1. Sketch showing how the real FFO $I-V$ curve (thick full line) may be constructed if one knew the $I-V$ curves of the ideal ("bare") junction, here represented by the three thin curves corresponding to three different control line currents $\left(i_{c l 1}>i_{c l 2}>i_{c l 3}\right)$. A positive sign of $K=\sigma / \beta$ (see Eq. (13)) is used, so that the magnetic field from the bias current makes the measured $I-V$ curve steeper. For large $K$-values the $I-V$ curve may have a measured dynamic resistance $R_{d}^{\prime} \leq 0$.

increment the two currents by $\Delta I$ and $\Delta I_{c l}$, respectively. The voltage change $\Delta V$ is determined by measuring the frequency shift of the emitted radiation. Since the uncertainty of the incremental currents $\Delta I$ and $\Delta I_{c l}$ can be reduced by averaging, the uncertainty of the dynamic resistances is dominated by the voltage (frequency) measurement uncertainty, which is less than $\sim 5 \mathrm{nV}$ (corresponding to $\sim 10 \mathrm{MHz}$ ). On the steep structures (Fiske steps) in the $I-V$ curve the free-running linewidth is of the order $100 \mathrm{kHz}$.

As mentioned above the $I-V$ curve (7) of the "bare" junction depends on the distribution of the junction current $i-$ i.e. on the dependence of $\eta(x)$ on $x$. Over the years several attempts have been made to reduce the linewidth by modifying the physical shape of the junction and of the superconducting electrodes near the junction, e.g. using a so-called "unbiased tail" as modeled by various current distributions [14]-[16]. From (14) and Fig. 1 it is obvious that a small value of the measured dynamic resistance $R_{d}^{\prime}$ in a real FFO not necessarily implies a narrow linewidth. This is only true for the ideal ("bare") junction with $K=0$. This mistake seems to have been carried over from the lumped junction scenario for over twenty years.

Looking at Fig. 1 one can obtain a situation where the measured FFO $I-V$ curve has a negative value of the dynamic resistance $R_{d}^{\prime}$. Such "back-bending" has been observed both experimentally and in our numerical simulations based on the sine-Gordon model. In a bias point where $R_{d}^{\prime}=0$ the measured FFO linewidth is solely due to internal bias current fluctuations conveyed via the $K$ factor.

\section{IV. $K$-VAlues For Different Bias Geometries}

Recently, linewidth measurements as described above have also been performed on a given FFO subjected to different bias configurations in order to explore the corresponding $K$-values. On the test chip we have a tri-layer $\mathrm{Nb}-\mathrm{AlOx}-\mathrm{Nb}$ structure with accessible contact pads. In the usual bias configuration (near-

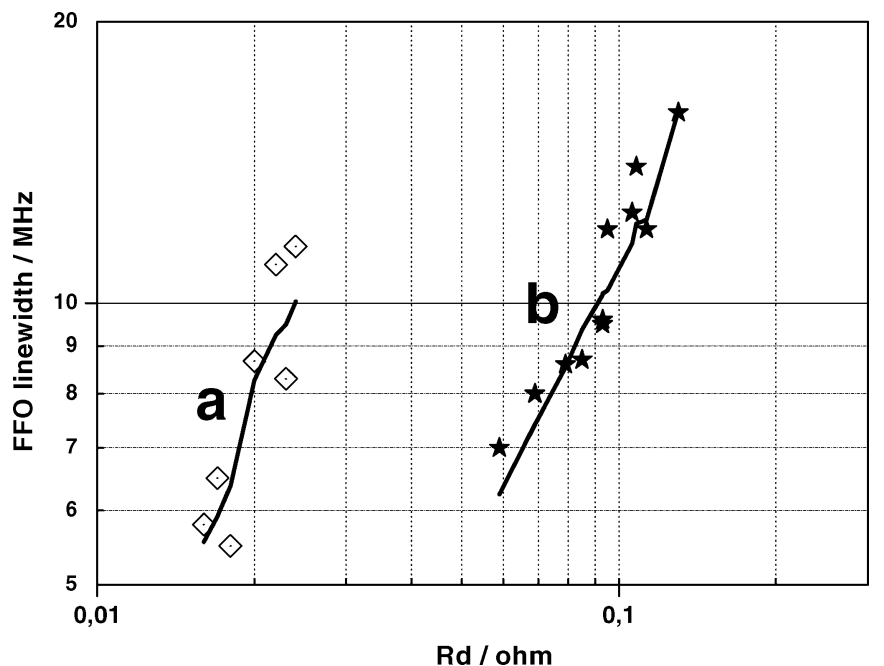

Fig. 2. Measured linewidth versus dynamic resistance for a given FFO with fixed bias current in two different bias configurations a) and b) (see text). The best fit to Eq. (14) (full curve) is obtained with $K=+0.25$ and $K=-1.1$, respectively. Frequency range $490-710 \mathrm{GHz} . T=4.2 \mathrm{~K}$.

overlap geometry) the DC bias current $I$ is supplied through the junction from the bottom $\mathrm{Nb}$-film to the top $\mathrm{Nb}$-film, and the control current $I_{c l}$ (flowing in the bottom Nb-film) generates a magnetic field which is dominantly perpendicular to the long junction dimension. In this bias configuration the flux flow is in the $I_{c l}$ direction and $K \approx 0$ yields the best fit to (14). If we maintain the $I_{c l}$ flowing in the bottom film there are two other ways to supply the bias current $I$. Either we can connect the input DC bias lead to the $I_{c l}$ output pad (configuration a) or to the $I_{c l}$ input pad (configuration b) and thus obtain two radically different bias geometries (see the ideal examples below). As shown in Fig. 2 the best fit to (14) in configuration a) is obtained for $K=+0.25$ while in configuration $\mathrm{b}$ ) we get the best fit for $K=-1.1$. The dependence of the $K$-values on the geometry supports our simple theory. Here we have only presented typical results measured for fixed bias current in the frequency range $490-710 \mathrm{GHz}$ at $T=4.2 \mathrm{~K}$ on a single FFO junction having $R_{n} S=37 \Omega(\mu \mathrm{m})^{2}$ (product of normal state resistance and junction area). An extensive study where also an extra external magnetic bias current may be supplied from additional superconducting control lines will be published elsewhere. These measurements indicate that a more elaborate theory is needed in order to explain the complicated dynamics in FFO's with smaller $R_{n} S$ values.

\section{General Case, Ideal Examples}

In general for a given geometry we can write

$$
\begin{aligned}
& w \kappa_{1}=\sigma_{1} i+\beta_{1} i_{c l} \\
& w \kappa_{2}=\sigma_{2} i+\beta_{2} i_{c l} \\
& \eta w l=\sigma_{3} i+\beta_{3} i_{c l} .
\end{aligned}
$$

From (4) we get $\sigma_{2}-\sigma_{1}+\sigma_{3}=1$ and $\beta_{2}-\beta_{1}+\beta_{3}=0$ where $\sigma_{2}-\sigma_{1}$ is just the inline fraction $1-\chi$ of the junction current and $\sigma_{3}$ is the overlap fraction $\chi$. From (5) we get

$$
\kappa=\frac{\sigma_{1}+\sigma_{2}}{2} i+\frac{\beta_{1}+\beta_{2}}{2} i_{c l} .
$$




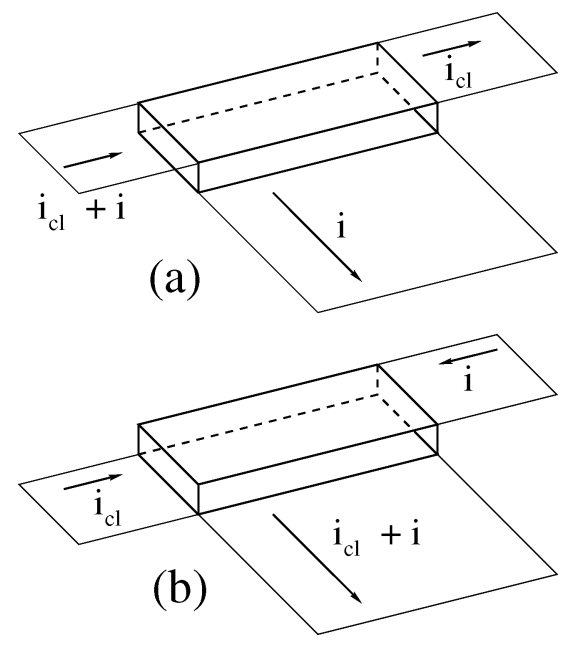

Fig. 3. Illustration of example 2, half inline; (a) $K=1 / 2, \chi=1 / 2$. (b) $K=$ $1, \chi=1 / 2$. The figure is not to scale. In the superconducting thin-films currents flow with equal magnitude in top and bottom of the film.

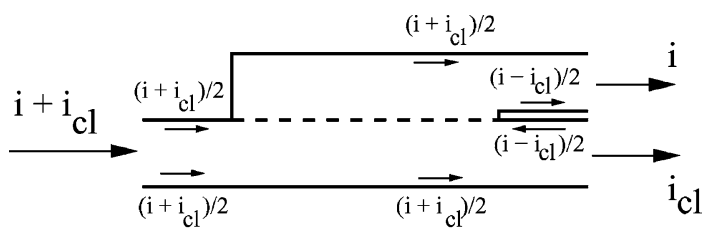

(a)

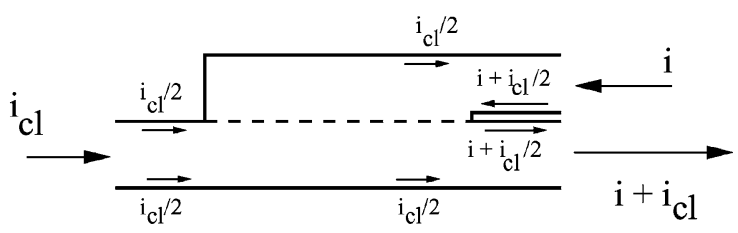

(b)

Fig. 4. Illustration to example 3. Pure inline $(\chi=0)$. The figure is not to scale. The total current in each superconducting thin-film is the sum of the currents flowing in the top and bottom of the film as indicated. The dashed line indicates the tunnel barrier. Two cases; a) $\sigma=0$ and therefore $K=0$ and b) $\sigma=1 / 2$, $\beta=1 / 2$ and therefore $K=1$.

This should be identical to (9) therefore we have

$$
-\sigma=\frac{\left(\sigma_{1}+\sigma_{2}\right)}{2} \text { and } \beta=\frac{\left(\beta_{1}+\beta_{2}\right)}{2}
$$

It is clear that $\sigma$ can be ascribed to an asymmetric feed of the junction. $K=\sigma / \beta=1$ means that the bias current $i$ and the control line current $i_{c l}$ (if equal) produce the same magnetic field. The situation is illustrated by three (ideal) examples which can be analyzed analytically. We assume that the external currents $i$ and $i_{c l}$ follow the same path on one side of the tunnel junction, that the magnetic field along every edge of the oxide layer is constant, and that the superconducting electrodes (Nb-films) are much thicker than the London penetration depth so currents flow in a very thin layer in the top and bottom of the films.

1) Pure overlap. If the bias current $i$ is purely overlap $(\chi=$ 1 ) there is no asymmetry in the bias current, therefore $\sigma=0$ and $K=0$.
2) Half inline. In the half inline case $(\chi=1 / 2)$ there are two different cases. 2a) The first situation is depicted in Fig. 3(a). Simple considerations give

$\sigma_{2} \simeq \sigma_{3} \simeq \frac{1}{2}, \quad \beta_{2}=\beta_{1}=\frac{1}{2}, \quad$ and $\quad \sigma_{1} \simeq \beta_{3} \simeq 0$ or $\beta=1 / 2$ and $\sigma=1 / 4$ and therefore $K=1 / 2$. 2b) The other situation with half inline is shown in Fig. 3(b). Here

$$
\sigma_{2} \simeq \sigma_{3} \simeq \beta_{1} \simeq \beta_{3} \simeq \frac{1}{2} \text { and } \sigma_{1} \simeq \beta_{2} \simeq 0
$$

or $\beta=1 / 4$ and $\sigma=1 / 4$ and therefore $K=1$.

3) Pure inline. If the bias current is purely inline $(\chi=0)$ there are two cases. Let $i_{c l}$ flow in the bottom film. If $i$ flows into one end of the junction from the bottom film and leaves the junction through the top film and the other end of the junction (Fig. 4(a)) there is no asymmetry in the current, $\beta=1 / 2$ therefore $\sigma=0$ and $K=0$. If the bias current $i$ leaves the junction from the same end as it enters (Fig. 4(b)) the asymmetry in the current is $\sigma=1 / 2$, $\beta=1 / 2$ and therefore $K=1$.

\section{ACKNOWLEDGMENT}

The authors would like to thank C. Mahaini for collaboration in the early stages of the project.

\section{REFERENCES}

[1] D. Rogovin and D. J. Scalapino, "Fluctuation phenomena in tunnel junctions," Ann. Phys., vol. 86, pp. 1-90, 1974.

[2] A. Zorin, "Fluctuations in a Josephson junction with arbitrary bias," Physica B, vol. 108, pp. 1293-1299, 1981.

[3] E. Jørgensen, V. P. Koshelets, R. Monaco, J. Mygind, M. R. Samuelsen, and M. Salerno, "Thermal fluctuations in resonant motion of fluxons on a Josephson transmission line: theory and experiment," Phys. Rev. Lett., vol. 49, pp. 1093-1096, 1982

[4] M. Salerno, M. Samuelsen, and A. Yulin, "Spectral linewidth of Josephson oscillators," Phys. Rev. Lett., vol. 86, pp. 5497-5499, 2001.

[5] A. Betenev and V. Kurin, "Radiation spectrum of a long Josephson flux-flow oscillator," Phys. Rev. B, vol. 56, pp. 7855-7857, 1997.

[6] A. Ustinov, H. Kohlstedt, and P. Henne, "Giant radiation linewidth of multifluxon states in long Josephson junctions," Phys. Rev. B, vol. 77, pp. 3617-3624, 1996.

[7] A. Golubov, B. Malomed, and A. Ustinov, "Radiation linewidth of a long Josephson junction in the flux-flow regime," Phys. Rev. Lett., vol. 54, pp. 3047-3050, 1996.

[8] V. P. Koshelets, S. V. Shitov, A. V. Shchukin, L. V. Filippenko, J. Mygind, and A. V. Ustinov, "Self-pumping effects and radiation linewidth of Josephson flux-flow oscillators," Phys. Rev. B, vol. 56, pp. 5572-5577, 1997.

[9] V. Koshelets, P. D. A. Ermakov, A. Sobolev, A. Baryshev, P. Wesselius, and J. Mygind, "Radiation linewidth of flux flow oscillators," Supercond. Sci. Tech., vol. 14, pp. 1041-1045, 2001.

[10] A. L. Pankratov, "Form and width of spectral line of a Josephson flux flow oscillator," Phys. Rev. B, vol. 65, 2002. pp. 054 504-(1-9).

[11] V. P. Koshelets, A. Shchukin, I. L. Lapytskaya, and J. Mygind, "Spectral linewidth of autonomous and injection-locked flux-flow oscillators,' Rev. Phys. B, vol. 51, pp. 6536-6541, 1995.

[12] M. R. Samuelsen and S. A. Vasenko, "Influence of the bias current distribution on the static and dynamic properties of long Josephson junctions," J. Appl. Phys., vol. 57, pp. 110-112, 1985.

[13] M. Cirillo, N. Grønbech-Jensen, M. Samuelsen, M. Salerno, and G. V. Rinati, "Fiske modes and Eck steps in long Josephson junctions: theory and experiments," Phys. Rev. B, vol. 58, pp. 12 377-12 384, 1998.

[14] T. Nagatsuma, K. Enpuku, F. Irie, and K. Yoshida, "Flux flow type Josephson oscillator for millimeter and submillimeter wave region," $J$ Appl. Phys., vol. 63, pp. 1130-1138, 1988.

[15] Y. M. Zhang and P. Wu, "Numerical calculation of the height of velocitymatching steps of flux flow type Josephson oscillator," J. Appl. Phys., vol. 68, pp. 4703-4709, 1990.

[16] A. L. Pankratov, "Long Josephson junction with spatially inhomogeneous driving," Phys. Rev. B, vol. 66, p. 134 526, 2002. 